CASE REPORT

\title{
Groin pain in sportsmen is not always musculotendinous in origin
}

\author{
K F Gomez, R Dawson, S G Davies, M E Foster
}

Br J Sports Med 2002;36:470-472

The case is reported of a young rugby player who presented with pubic tenderness not caused by any muscular injury or direct trauma but by a retropubic abscess.
A cute or subacute groin pain is a common condition in sportsmen. Most are musculotendinous strains or tears that readily settle with a combination of rest, analgesia, and physiotherapy. However, not all of them are. We report here a case of a young rugby player who presented with pubic tenderness not from any muscular injury or direct trauma, but from a retropubic abscess.

\section{CASE REPORT}

A 21 year old rugby player presented with bilateral suprapubic tenderness of 10 days duration. It was first noticed after a training session, and despite analgesics and rest had not settled. The term "suprapubic" in this case refers to the region just superior to the pubic symphysis and including the origin of the adductor longus bilaterally.

The patient had suffered a similar episode six months previously when he slipped on some wet grass and hyperabducted both hips. He was seen at a private rehabilitation centre where he was diagnosed with a muscular haematoma secondary to trauma. He then had aggressive physiotherapy. Unfortunately, his pain did not improve and he continued to complain of severe suprapubic tenderness, even on minimal exertion. He presented to the accident and emergency department of his local hospital with these symptoms some days after the initial injury. A thorough examination was performed by a casualty officer, followed by a plain anteroposterior radiograph of his hips. This showed no bony injury and therefore the patient was prescribed analgesics and given bilateral injections of DepoMarcaine into both groins at the insertion of the adductors - that is, the pubic tubercle-as his pain was localised to this area. He obtained relief from the injected steroid preparation and returned to his usual daily activities within a few days.

He presented to the same hospital six months later with an acute onset of localised tenderness at the origins of the adductor longus bilaterally. Movement of the left hip was restricted, especially at the extremes of abduction and external rotation, and he found difficulty in weight bearing. He was apyrexial at the time of admission and all initial blood results were normal. The next day, however, the patient developed a pyrexia of $38.5^{\circ} \mathrm{C}$. His erythrocyte sedimentation rate was $75 \mathrm{~mm} /$ hour in the first hour (normal range for men: 1-13 mm/hour), and his C reactive protein level was $229 \mathrm{mg} / \mathrm{l}$ (normal range: $<10 \mathrm{mg} / \mathrm{l}$ ). Mid stream urine samples showed 68 white blood cells, 50-100 red blood cells, and some mixed contaminants. Blood cultures showed a heavy growth of

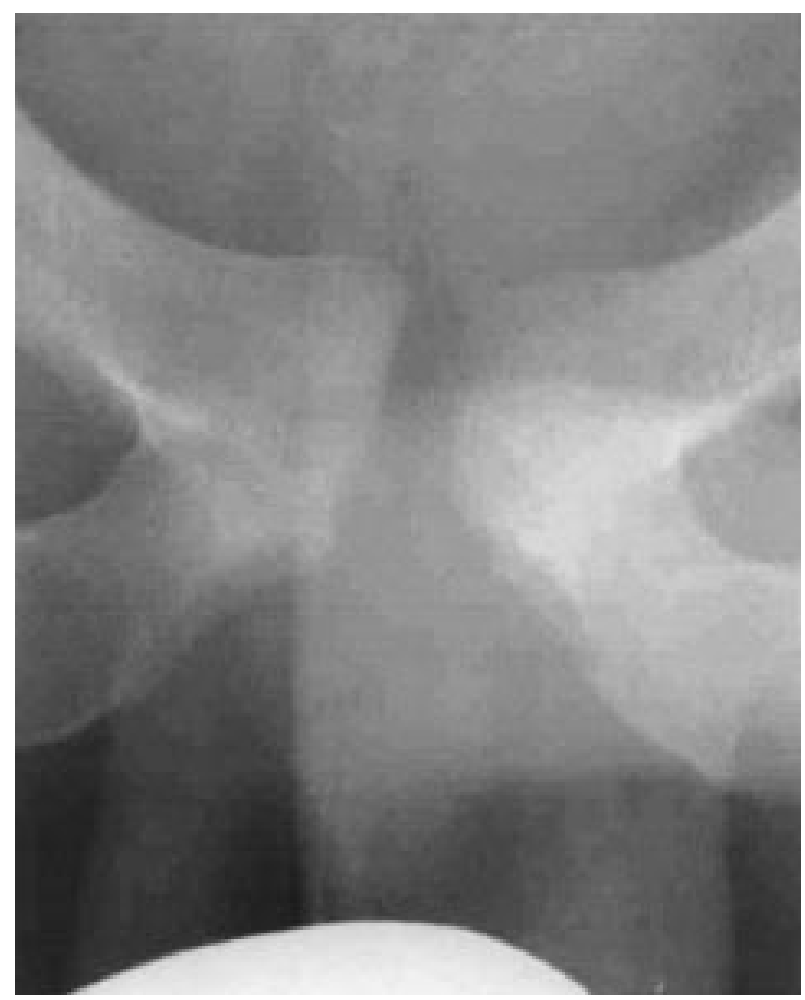

Figure 1 Plain radiograph of the pelvis showing an avulsion injury to the left inferior aspect of the pubic symphysis.

Staphylococcus aureus sensitive to flucloxacillin. He responded well to intravenous antibiotics and his symptoms settled.

\section{RADIOLOGICAL INVESTIGATIONS}

To find the source of the patient's infection, a number of radiological investigations were performed. Plain radiographs of the pelvis showed an avulsion injury to the left inferior aspect of the pubic symphysis (fig l). An isotope bone scan showed intense uptake in the symphysis pubis bilaterally, which was interpreted by a consultant radiologist as an active osteitis pubis. In view of the history of pyrexia and limitation of left hip movement, magnetic resonance imaging (MRI) was performed. This showed a large retropubic abscess anterior to the bladder, marrow oedema in the symphysis pubis, and extensive signal abnormality in the left and right

Abbreviations: $M R I$, magnetic resonance imaging; $C T$, computed tomography 


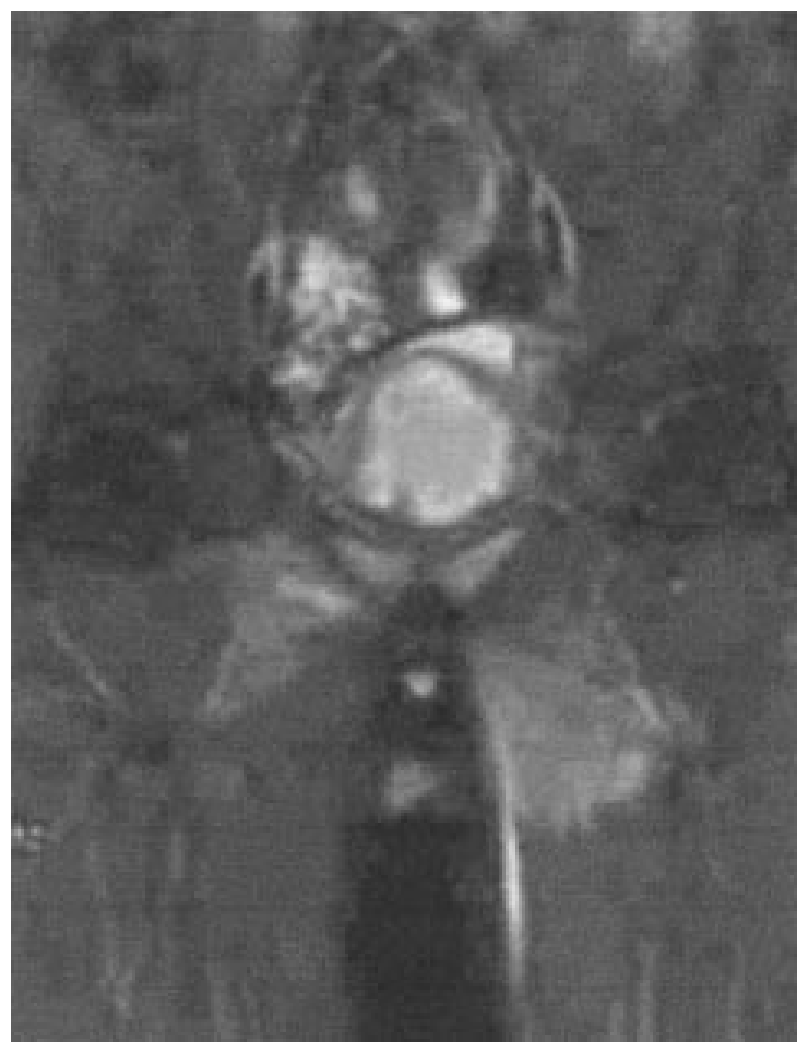

Figure 2 Coronal magnetic resonance imaging scan of the pelvis showing an abscess and abnormal muscle.



Figure 3 Computed tomography guided abscess drainage.

adductor musculature, suggesting tracking of the abscess (fig 2). Computed tomography (CT) guided drainage of the retropubic abscess was performed. Aspiration yielded $50 \mathrm{ml}$ of thick pus (fig 3). A follow up MRI scan a few weeks later showed resolving local inflammatory change in the presence of a small residual abscess in an otherwise asymptomatic patient.

\section{DISCUSSION}

Pain is a common symptom in many sportsmen who undergo rigorous training. The reasons are legion and must be directed

\section{Take home message}

We recommend that all sportsmen presenting with "activity related groin pain" should have a mid stream urine sample analysed before being discharged.

on the basis of clinical findings at the time of presentation. Osteitis pubis may present as pubic pain in the presence of a low grade pyrexia. Clinically therefore it is extremely difficult to distinguish between osteitis pubis and an underlying osteomyelitis. ${ }^{1}$ Fortunately pyogenic infection of the symphysis pubis is uncommon. ${ }^{2} \mathrm{~A}$ few series have reported these findings in diabetics, ${ }^{34}$ intravenous drug abusers, ${ }^{5}$ and patients recently undergoing urological intervention. Plain radiographs are of little benefit in the acute stage as changes of rarefaction and periosteal elevation are usually delayed. Therefore treatment cannot be based solely on radiological criteria. $^{2}$

The treatment for osteitis pubis is a combination of rest and/or modification of extreme activity in the case of sportsmen, oral anti-inflammatory analgesia, and hip stretching exercises. This management strategy may not be amenable to some sportsmen, who undergo a combination of corticosteroid and local anaesthetic injections to hasten recovery. ${ }^{6}$ All forms of injections carry their own risks, including introduction of infection, haemorrhage, and inadvertent intravascular injection with its subsequent cardiac side effects. Their use in the acute situation should therefore be limited. Intramuscular abscesses secondary to injections have also been reported. ${ }^{7}$

Bacteriuria is an uncommon finding in men, affecting only $0.5-4 \%$ of the population and usually confined to the elderly. ${ }^{8}$ Pyuria may be an indicator of urinary infection if supportive evidence exists, but may equally represent an inflammatory process occurring elsewhere. If pyuria is present therefore, further investigation is warranted. Ultrasound of the renal tract and bladder is an easy investigation that may aid in the diagnostic process. Sterile pyuria, however, should always raise the possibility of a tuberculous infection of the renal tract. $^{8}$

Not all sportsmen presenting with groin pain will have such significant pathology. We know of only one other similar case report, where an obturator internus abscess was found in a 19 year old footballer who presented with pubic pain. ${ }^{9}$ However, this case does highlight two underlying lessons to be learnt. The first is that "groin pain" in a sportsman does not always have a musculotendinous cause, and the second is that treating acute muscular strains with corticosteroid and local anaesthetic injections alone should not be undertaken lightly. Awareness of these facts in combination with sound clinical judgment will ensure cases such as this remain a rarity.

\section{Authors' affiliations}

K F Gomez, R Dawson, S G Davies, M E Foster, Royal Glamorgan Hospital, Ynysmaerdy, Llantrisant CF72 8XR, Wales, UK

Correspondence to: Mr Gomez, Department of Surgery, Royal Glamorgan Hospital, Llantrisant, CF72 8XR, Wales, UK; kfg@doctors.org.uk

Accepted 17 April 2002

\section{REFERENCES}

1 Fricker PA, Taunton JE, Ammann W. Osteitis pubis in athletes. Sports Med 1991;12:266-79.

2 Burns JR, Gregory JG. Osteomyelitis of the pubic symphysis after urologic surgery. J Urol 1977;1 18:803-5. 
3 Sequeira $\mathbf{W}$, Jones $E$, Siegel ME, et al. Pyogenic infections of the pubic symphysis. Ann Intern Med 1982;96:604-6.

4 Takahashi M, Miyake H, Iwase M, et al. Pyogenic infection of the pubic symphysis in a diabetic patient. Scand J Infect Dis 1999;31:325-6.

5 Kido D, Bryan D, Halpern M. Hematogenous osteomyelitis in drug addicts. AJR Am J Roentgenol 1973;118:356-63.

6 Holt MA, Keene JS, Graf BK, et al. Treatment of osteitis pubis in athletes. Results of corticosteroid injections. Am J Sports Med 1995;23:601-6.
7 Rygnestad T, Kvam AM. Streptococcal myositis and tissue necrosis with intramuscular administration of diclofenac (Voltaren). Acta Anaesthesiol Scand 1995;39:1 128-30

8 James MJ. Investigation of the lower urinary tract. Surgery

1996;14:203-6.

9 Guis-Sabatier S, Pieri-Balandraud N, Garnier-Soumet P, et al. Pubic pain in athletes: a case due to an abscess in the obturator muscle. Rev Rhum Engl Ed 1999;66:58-60 